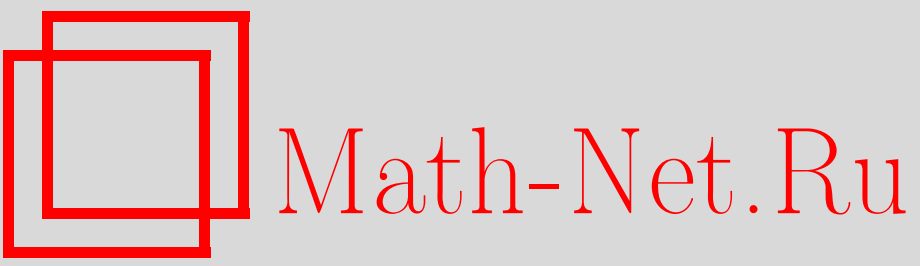

В. Н. Соловьев, О субдифференциале максимума семейства выпуклых функций, УМH, 1996, том 51, выпуск 2, 175-176

DOI: https: //doi.org/10.4213/rm963

Использование Общероссийского математического портала Math-Net.Ru подразумевает, что вы прочитали и согласны с пользовательским соглашением

http://www.mathnet.ru/rus/agreement

Параметры загрузки:

IP : 3.85 .183 .62

26 апреля 2023 г., 14:12:59 


\title{
О СУБДИФФЕРЕНЦИАЛЕ МАКСИМУМА СЕМЕЙСТВА ВЫПУКЛЫХ ФУНКЦИЙ
}

\author{
В. Н. СОЛОвьЕВ
}

Пусть $f_{s}(x): X \rightarrow \overline{\mathbb{R}}=\mathbb{R}^{1} \cup\{+\infty\}$ - выпуклые функции, определенные на хаусдорфовом локально выпуклом линейном топологическом пространстве $X, S$-хаусдорфово топологическое пространство, и

$$
f(x):=\sup _{s \in S} f_{s}(x), \quad S(x):=\left\{s \in S \mid f_{s}(x)=f(x)\right\} .
$$

Если $X^{*}$ - множество всех линейных непрерьвных функционалов $\left\langle x^{*}, x\right\rangle$ на $X$, то множество $\partial f\left(x_{0}\right):=\left\{x^{*} \in X^{*} \mid f(x) \geqslant f\left(x_{0}\right)+\left\langle x^{*}, x-x_{0}\right\rangle, x \in X\right\}$ называется субдифференциалом функции $f(x)$ в точке $x_{0}$ [4]. Обозначим через $\operatorname{dom} f$ множество всех точек $x \in X$, где функция $f(x)$ конечна, и через $N\left(x_{0} \mid \operatorname{dom} f\right):=\left\{x^{*} \in X^{*} \mid\left\langle x^{*}, x-x_{0}\right\rangle \leqslant 0, x \in \operatorname{dom} f\right\}$ - нормальный конус этого множества в точке $x_{0} \in \operatorname{dom} f$.

В работе вычислены субдифференциалы функции $f(x)$ без предположения о компактности пространства $S$.

Теорема 1. Пусть выполнены следующие условия:

(1) многозначное отображсение $S(x): \operatorname{dom} f \rightarrow S$ имеет непустые компактные значения и полунепрерывно сверху на любом отрезке в $\operatorname{dom} f$;

(2) функции $f_{s}(x)$ полунепрерывны сверху по s на $S \forall x \in \operatorname{dom} f$;

(3) функиии $f_{s}(x), s \in S$, непрерывны в некоторой точке $x_{1} \in \operatorname{int} \operatorname{dom} f$. Тогда при всех $x_{0} \in \operatorname{dom} f$

$$
\partial f\left(x_{0}\right)=\overline{\operatorname{conv} \bigcup_{s \in S\left(x_{0}\right)} \partial f_{s}\left(x_{0}\right)+N\left(x_{0} \mid \operatorname{dom} f\right)},
$$

где замыкание берется в слабой *-топологии пространства $X^{*}$.

Формула (4) аналогична известной теореме о субдифференциале максимума выпуклых функций, полученной в [1] для компактного множества индексов $S$. Для конечного множества индексов $S$ окончательный результат получен в $[6]$.

Теорема 2. Пусть $X=\mathbb{R}^{n}$ и выполнены условия (1)-(3), а также

(5) найдется точка $x_{1} \in \operatorname{ridom} f_{s}, s \in S, x_{1} \in \operatorname{ridom} f$.

Тогда при всех $x_{0} \in \operatorname{dom} f$ имеет место равенство

$$
\begin{aligned}
\partial f\left(x_{0}\right)=\left\{\sum_{j=1}^{n+1} \alpha_{j} x_{j}^{*}+x_{0}^{*} \mid\right. & x_{j}^{*} \in \partial f_{s_{j}}\left(x_{0}\right), s_{j} \in S\left(x_{0}\right), \alpha_{j} \geqslant 0, \\
& \left.j=1, \ldots, n+1, x_{0}^{*} \in N\left(x_{0} \mid \operatorname{dom} f\right), \sum_{j=1}^{n+1} \alpha_{j}=1\right\} .
\end{aligned}
$$

СлЕДСТвИЕ. Пусть функции $f_{s}(x)$ вогнуты по $s$ на выпуклом множестве $S\left(x_{0}\right)$ nри всех $x \in \operatorname{dom} f$ и удовлетворяют условиям теорем 1 или 2. Тогда при $x_{0} \in \operatorname{dom} f$ имеет место равенство

$$
\partial f\left(x_{0}\right)=\bigcup_{s \in S\left(x_{0}\right)} \partial f_{s}\left(x_{0}\right)+N\left(x_{0} \mid \operatorname{dom} f\right)
$$


Теорема 3. Пусть $S$ - выпуклое подмножество рефлексивного банахова пространства $Y$, а функция $f_{S}(x): X \rightarrow \overline{\mathbb{R}}$ вогнута по $s$ на множестве $S$ при $x \in X$, удовлетворяет условиям (2), (3) и коэрцитивна по $s$ в точке $x_{0} \in \operatorname{dom} f$, m.e.

$$
\lim f\left(s, x_{0}\right)=-\infty \text { nрu } s \in S, s \rightarrow s_{0} \notin S, \text { либо при } s \in S,\|s\| \rightarrow \infty
$$

(в условиях (2) и (8) множество $S$ снабжается относительной топологией, индуцируемой на $S$ из $Y)$. Тогда в этой точке справедлива формула (7).

Теорема 4. Пусть мнохсество $S$ относительно компактно в хаусдорфовом топологическом пространстве $Y$, а функиия $f_{S}(x): X \rightarrow \overline{\mathbb{R}}$ удовлетворяет условиям (2), (3) u (8) в точке $x_{0} \in \operatorname{dom} f$. Тогда в әтой точке справедлива формула (4).

Далее $S$ - произвольное множество индексов.

Теорема 5. Пусть $f_{s}(x): \mathbb{R}^{n} \rightarrow \overline{\mathbb{R}}, s \in S$, - произвольное семейство выпуклых функчий, и пусть $\varphi_{s}(x):=f_{s}(x), x \in \operatorname{dom} f, \varphi_{s}(x):=+\infty$, иначе. Предположим, что нижняя грань $\inf _{s \in S} \varphi_{s}^{*}\left(x^{*}\right)$ достигается всюду, где она конечна, и полунепрерывна снизу на $\mathbb{R}^{n}$ (как функция переменной $\left.x^{*}\right)$. Тогда при всех $x_{0} \in \operatorname{dom} f$ имеет место равенство

$$
\partial f\left(x_{0}\right)=\left\{\sum_{j=1}^{n+1} \alpha_{j} x_{j}^{*} \mid x_{j}^{*} \in \partial \varphi_{s_{j}}\left(x_{0}\right), s_{j} \in S\left(x_{0}\right), \alpha_{j} \geqslant 0, \sum_{j=1}^{n+1} \alpha_{j}=1\right\} .
$$

Условия теоремы 5 выполняются в том случае, когда все функции $f_{s}(x)$ полунепрерывны сверху по $s$ на компакте $S$ при любом $x \in \operatorname{dom} f$ и имеют общую область определения $\operatorname{dom} f_{s}=$ $\operatorname{dom} f:=M, s \in S$. В такой форме (при дополнительных условиях замкнутости множества $M$ и полунепрерывности снизу функций $f_{s}(x), s \in S$, на этом множестве) формула (9) получена в [2], [3].

ТеОрема 6. Пусть $f_{s}(x): X \rightarrow \overline{\mathbb{R}}, s \in S,-$ произвольное семейство полунепрерывньх снизу выпукльх функций, и пусть множсество $S(x)$ не пусто при всех $x \in \operatorname{dom} f$. Тогда следующие свойства әквивалентны:

a) $\partial f(x)=\bigcup_{s \in S(x)} \partial f_{s}(x)$ nри всех $x \in \operatorname{dom} f$;

b) множество $S^{*}\left(x^{*}\right):=\left\{s \in S \mid f_{s}^{*}\left(x^{*}\right)=f^{*}\left(x^{*}\right)\right\}$ не пусто при всех $x^{*} \in \operatorname{dom}\left(\partial f^{*}\right)$

c) множество $S(x) \cap S^{*}\left(x^{*}\right)$ не пусто для всех $x^{*} \in \partial f(x)$;

d) если $x^{*} \in \partial f(x)$, то $x^{*} \in \partial f_{s}(x)$ для некоторого $s \in S(x) \cap S^{*}\left(x^{*}\right)$;

е) если $x \in \partial f^{*}\left(x^{*}\right)$, то $x \in \partial f_{s}^{*}\left(x^{*}\right)$ для некоторого $s \in S(x) \cap S^{*}\left(x^{*}\right)$.

В этом случае

$$
f^{*}\left(x^{*}\right)=\min _{s \in S} f_{s}^{*}\left(x^{*}\right), \quad x^{*} \in \operatorname{dom}\left(\partial f^{*}\right) .
$$

В [5] дан содержательный пример применения равенства (10).

\section{СПИСОК ЛИТЕРАТУРЫ}

[1] Иоффе А. Д., Тихомиров В.М. Теория экстремальных задач. М.: Наука, 1974. [2] Левин В. Л. // Матем. сб. 1969. Т. 79. № 2. С. 250-263. [3] Левин В. Л. Выпуклый анализ в пространствах измеримых функций. М.: Наука, 1985. [4] Рокафеллар Р. Т. Выпуклый анализ. М.: Мир, 1973. [5] Соловьев В. Н. // Anal. Math. 1993. V. 19. № 4. P. 297-315. [6] Volle M. // C. R. A. Sci. Paris. 1993. V. 317. Serie I. P. 845-849. [7] Hiriart-Urruty J.-B., Moussaoui M., Seeger A., Volle M. // Non-linear Analysis, Theory, Methods and Applications. 1995. V. 24. № 12. P. 1727-1754. 